\title{
REVIEW
}

\section{Asthma and suicide-related adverse events: a review of observational studies}

\author{
N. lessa*, M.L. Murray*,\#, S. Curran ${ }^{\star}$ and I.C.K. Wong ${ }^{\star, \#,+}$
}

ABSTRACT: Suicide is a major public health concern. There are several risk factors associated with suicide. Chronic illnesses, such as asthma, have been linked to an increased risk of suiciderelated events. This study reviews the evidence of an association between asthma and suicide using published epidemiological observational studies.

An electronic search using PubMed and EMBASE was performed. Studies that investigated the association of asthma with suicide-related behaviour were selected. Studies were examined to form a descriptive analysis.

Six observational studies met the selection criteria, of which at least one suicide-related adverse event was studied. Three studies investigated completed suicide, two suicide attempts and four suicide ideation. Two of the studies focused on individuals aged $<18$ yrs.

Evidence from observational data support the hypothesis of an association between asthma and suicide-related behaviour (ideation, attempts and completion); however, epidemiological studies, with more objective measures and larger sample sizes, adjusting for a wider scope of suicide-related confounding factors (e.g. comorbidities), and with a longitudinal design, are needed for a more conclusive answer.

KEYWORDS: Asthma, attempt, ideation, review, suicide

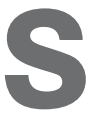
uicide is a major public health issue worldwide. The World Health Organization (WHO) reports that nearly one million people die due to suicide each year, with a global mortality rate of 16 deaths per 100,000 or one death every $40 \mathrm{~s}$. It is the 14th leading cause of death worldwide and accounts for $1.5 \%$ of all deaths [1]. The suicide rates in the UK in 2009 were recorded as 17.7 and 5.2 per 100,000 in males and females, respectively [2]. In England there is an average of one person dying every $2 \mathrm{~h}$ as a result of suicide and it is one of the top three leading causes of death in people aged 15-34 yrs in the UK [3].

In order to decrease the burden and prevent suicides there needs to be an understanding of factors that increase the risk of suicidal behaviour. Several risk factors exist including demographic, psychiatric, psychological and biological factors [4]. Increased suicidal thoughts and behaviour are more common in chronic physical illness, and increased suicidal ideation is associated with conditions such as asthma, even in the absence of depression [5-10].

Asthma is one of the most common chronic diseases among children, with approximately one in 11 children suffering from asthma in the UK [11]. On average, four people per day or one person every $7 \mathrm{~h}$ dies from asthma in the UK [12]. The WHO estimates that there are currently 300 million people suffering from asthma and asthma is also estimated to account for $\sim 250,000$ deaths per year worldwide [12, 13]. Asthma can have a significant effect on quality of life, and consequences include sleeplessness, daytime fatigue, reduced activity levels, school and work absenteeism, and mental health problems [14-16]. It is hypothesised that asthma is a risk factor associated with suicidal behaviour. The question of whether such an association exists is particularly important because of recent concerns about the possible effects of some types of asthma treatment on suicide risk [17, 18]. The aim of this article is to systematically search, identify and review evidence in the literature of an association between asthma and suicidal behaviour (suicide ideation, attempt and completion) in the general population.

\section{METHODS}

Two investigators (N. Iessa and M.L. Murray) carried out an electronic search of bibliographic

\section{AFFILIATIONS}

${ }^{*}$ Centre for Paediatric Pharmacy Research, The School of Pharmacy, University of London,

\# Institute of Child Health, University

College London, and

'Institute of Psychiatry, King's College London, London, UK.

+Dept of Pharmacology and

Pharmacy, Li Ka Shing Faculty of

Medicine, The University of Hong

Kong, Pokfulam, Hong Kong.

\section{CORRESPONDENCE}

I.C.K. Wong

Dept of Pharmacology and Pharmacy,

Li Ka Shing Faculty of Medicine

The University of Hong Kong

Pokfulam

Hong Kong

E-mail: wongick@hku.hk

Received:

June 082011

Accepted after revision:

June 242011

PROVENANCE

Submitted article, peer reviewed. 
databases. The search strategy included the following terms: Suicide OR suicidal OR self harm AND Asthma OR cough OR respiratory disease OR wheez* This strategy was inputted into PubMed (Medline 1966 to March 2011) EMBASE (1980 to March 2011) and International Pharmaceutical Abstracts (1970 to August 2010). No limits relating to language, age, type of article or date were imposed. The search was last updated on February 18, 2011. The study criteria included all epidemiological observational studies (cohort, cross-sectional and case controlled) for all population groups (adults and children). The outcome measures for suicidal behaviour were defined as suicide ideation, suicide attempts, self-injury and death by suicide. Studies that did not investigate asthma and suicide specifically were excluded.

There is some ambiguity with defining terms related to suicide and suicidal behaviour, making it difficult to compare studies and conduct a meta-analysis [19]. Most clinicians and researchers distinguish suicidal behaviour from non-suicidal self-injury, i.e. self-injury in which a person has no intention of dying. A suicide plan is the formulation of a specific method through which one intends to die and suicide attempt refers to engagement in potentially self-injurious behaviour in which there is at least some intent to die.

Articles generated from the search were combined using a reference tool (Reference Manager ${ }^{11.0}$; Thomson Researchsoft, New York, NY, USA). Duplicates were removed and titles and abstracts of articles were screened for relevance by two reviewers (N. Iessa and M.L. Murray). Potentially relevant articles were obtained in full and further screened to determine if they met inclusion criteria. Data from the articles were summarised in terms of study population, outcome measures, settings, and data collection and limitations to form a descriptive analysis.

\section{RESULTS}

After removal of duplicate articles a total of 803 articles were generated by the electronic search (fig. 1).

\section{Selection of studies}

After screening abstracts and titles, 11 articles were identified as potentially relevant and full texts were obtained. Three articles were excluded because they were not specific to asthma as an exposure $[7,8,20]$. One article was excluded as it did not measure suicide as an outcome and another article consisted of a series of cases where asthma was speculated to be a tool to commit suicide [21, 22]. Six articles met the inclusion criteria [5, 10, 23-26]. Table 1 summarises data extracted from the studies and compares methodology, outcomes and limitations of each of the studies.

\section{Summary of studies included}

\section{Study population}

Three studies were carried out in adults aged $>18$ yrs [5, 24, 25]. Two studies were conducted in youths aged 9-17 yrs [23] and 11-16 yrs [10], and one study with both adults and children [26].

\section{Outcome measures, measurements and data collection}

\section{Adults}

ClARKe et al. [24] list the two main outcomes of interest as suicide ideation and suicide ideation with suicide attempt. Other variables measured were socio-demographics and history

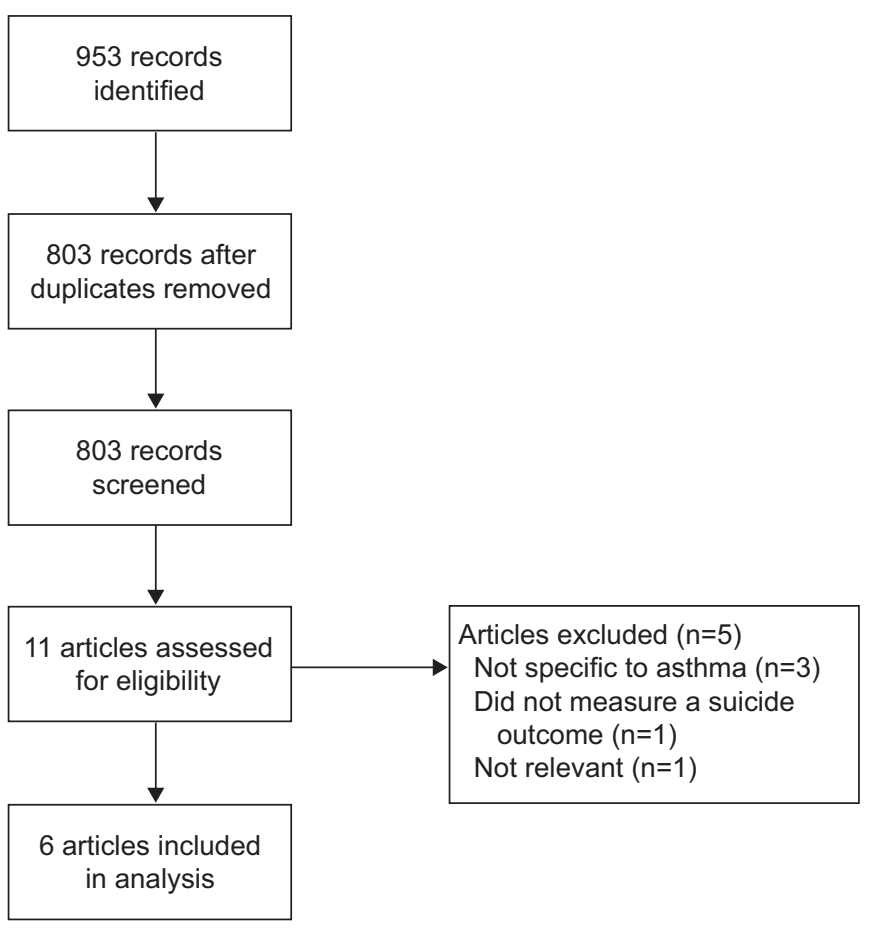

FIGURE 1. Results of electronic search.

of depression, anxiety, alcohol abuse and nicotine dependence. GOODWIN and EATON [5] used suicide ideation and attempts as primary outcomes at two time-points (waves) $1 \mathrm{yr}$ apart, followed by deaths due to suicide and depression at the third time-point at 15 yrs follow-up. In another study by GoODWIN et al. [25], the primary outcomes included suicide ideation as well as major depression, panic attacks, generalised anxiety disorder, alcohol and drug use disorder.

\section{Adults and youths}

The study conducted by QIN et al. [26] used completed suicide as an outcome measure obtained from the danish cause of death registry.

\section{Youths}

A study carried out in Taiwan by KuO et al. [10] had death due to suicide as its primary outcome. Other outcomes included natural death and cause of deaths. Suicide ideation and attempts were not measured. In a study by GoODWIN and MARUSIC [23], suicide ideation was the primary outcome assessed through questioning, other measures included; information on child psychiatric diagnosis; psychotropic medication use and mental health treatment; general functioning; family history of psychopathology; medication use and environmental risk and protective factors; physical health; social competence; educational aspiration; parental relationships; adverse life events; and socioeconomic status.

\section{Suicide ideation}

Suicide ideation was mainly assessed through interview questions. ClARKE et al. [24] determined suicide ideation through data obtained from the National Comorbidity Survey-Replication (NCS-R). The NCS-R survey was a national survey of people aged $\geqslant 18$ yrs carried out in 2001-2003. It was a fully structured 


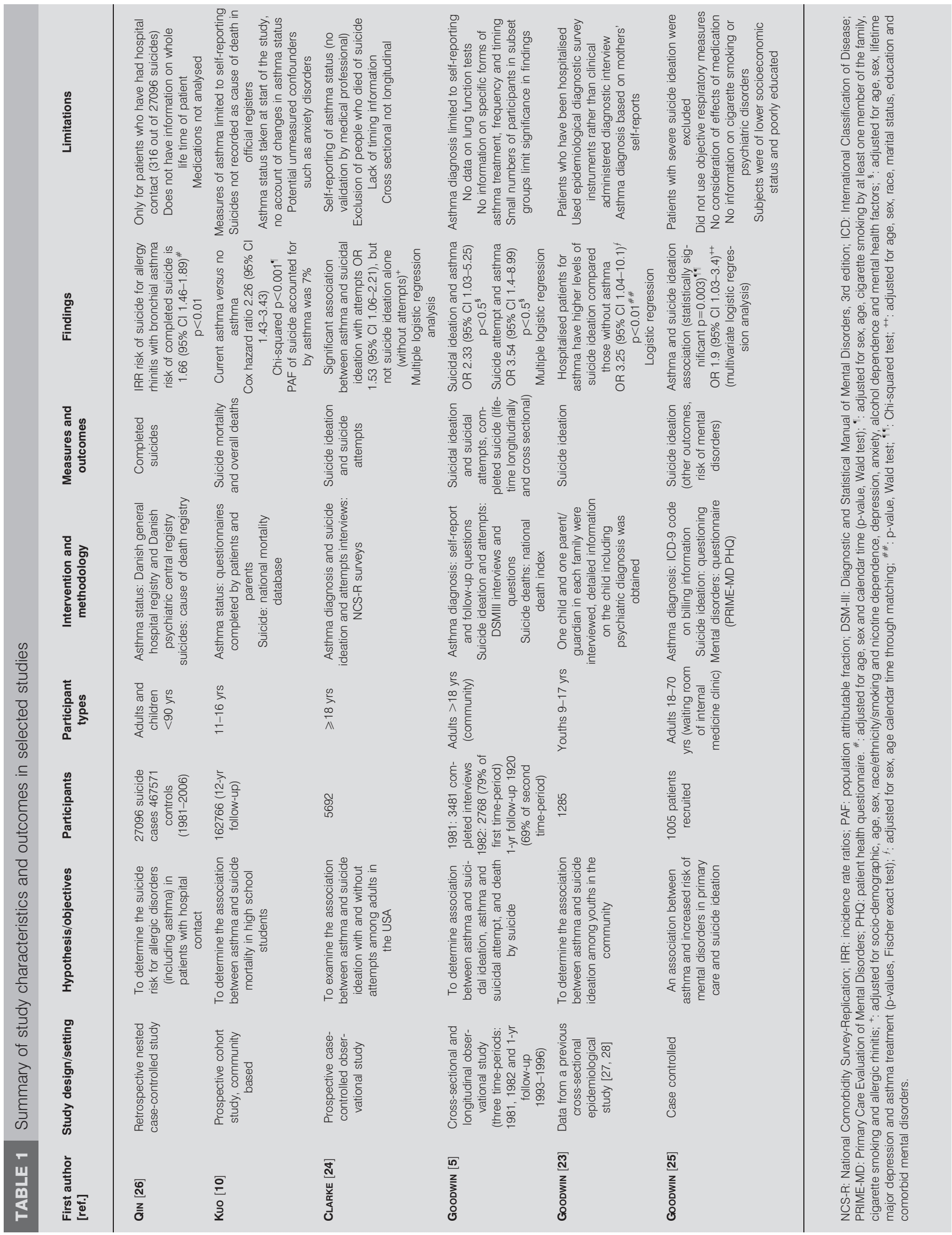


interview that was administered face to face by trained lay interviewers. It assessed 12-month occurrence of suicide ideation from baseline.

\section{Suicide attempt}

Two studies measured suicide attempt; GOODWIN and EATON [5] interviewed patients asking "have you ever attempted suicide?" and CLARKE et al. [24] used the NCS-R survey results.

\section{Completed suicide}

Three studies included completed suicide as an outcome measure. GOODWIN and EATON [5] linked patients to the National Death Index in the USA, KUO et al. [10] used the Department of health death certification system (National Mortality Database) in Taiwan and QIN et al. [26] identified deaths using the Danish cause of death registry.

\section{Setting and study design}

Five of the studies used large population data [5, 10, 24-26] and one recruited patients prospectively from a primary care clinic [23]. The majority of studies gave a cross-sectional overview with the exception of two studies. One study was based on the entire population to perform a nested case-control design [26] and the second observed suicide ideation, attempts and deaths longitudinally in addition to cross-sectionally [5].

Four out of the six studies were carried out in the USA [5, 23-25], one was carried out in Taiwan [10] and one in Denmark [26].

\section{Diagnosis of asthma}

GoODWIN et al. [25] used physician-based diagnosis obtained from billing records to identify patients with asthma. Other studies obtained information from the patients and/or parents of patients (among the youths) by means of interviews or through a video and written questionnaire. Examples of interview questions were as follows: "Have you ever had asthma?" and "Do you have asthma now?" [5]; "Did a doctor or other health professional ever tell you that you had asthma?" [24]; and "Has your child ever had asthma?" [22]. In the study by QIN et al. [26], the Danish General Hospital Registry was used to retrieve personal history of hospital contacts with allergic illness as a primary diagnosis. Under this umbrella, asthma codes were used to detect patients with allergic rhinitis and bronchial asthma [26].

\section{Risk factors for analysis}

All studies were adjusted for socio-demographic factors such as age, race, ethnicity and sex. Other comorbidities were also measured in different studies. CLARKE et al. [24] measured lifetime depression, anxiety, alcohol abuse, nicotine dependence and smoking status. GoODWIN and MARUSIC [23] measured child psychiatric diagnosis, psychotropic medication use and mental health treatment, general functioning, family history of psychotropic medication use and mental health treatment, family history of psychopathology environmental risk and protective factors. KuO et al. [10] measured cigarette smoking of family members and allergic rhinitis. The studies by GOODWIN and co-workers [5, 25] measured major depression and mental disorders, respectively. QIN et al. [26] measured whether patients had a history of mood disorder. The authors also made adjustments for mood disorders in allergic patients, but not for the strata of patients with asthma and allergy.

\section{Association between asthma and suicide}

All studies showed an association with the suicide-related outcome measured and asthma, even after adjusting for potential confounders. CLARKE et al. [24] showed that asthma was significantly $(p<0.001)$ associated with suicide ideation and attempts but not with suicide ideation alone. This remained statistically significant after adjusting for smoking, depression, panic disorder and alcohol abuse. In the study by GOODWIN and EATON [5], asthma significantly increased the odds of suicide ideation (OR 2.33, 95\% CI 1.03-5.25) and suicide attempt (OR 3.54, 95\% CI 1.4-8.99). Asthma increased the likelihood of suicidal ideation (OR 1.9, 95\% CI 1.03-3.4) after controlling for comorbid disorders in another study by GOODWIN et al. [25]. In youths, KuO et al. [10] showed that the incidence rate of suicide mortality in participants with current asthma to baseline was more than double that of those without asthma (11.0 versus 4.3 per 100,000 person-yrs). QIN et al. [26] showed an incidence rate ratio (IRR) of 1.66 (95\% CI 1.46-1.89) in those who that had allergy rhinitis with bronchial asthma and who were in-patients. However, outpatients did not show a significant IRR. GOODWIN and MARUSIC [23] showed that asthma was associated with a significantly increased likelihood of suicide ideation (OR 3.25, 95\% CI 1.04-10.1), compared with youths without asthma.

\section{DISCUSSION}

The studies suggest an association between asthma and suicide ideation and attempts in adults, suicide ideation in children, and completed suicide in both adults and children. This association remained evident after adjusting for socio-demographic factors in all studies. For studies that made adjustments for confounding by comorbidities such as major depression, mental disorders and environmental factors such as smoking, the association was reduced, but remained significant, indicating an independent association between asthma and suicidal behaviour.

The reasons for an association between suicidal behaviour and asthma are not clear. Possible factors include mood changes and anxiety that result from the disability and discomfort associated with asthma. Individuals might have frequent thoughts of death with increasing severity because they have a potentially life-threatening disease [14]. Other explanations include a link to the production of pro-inflammatory cytokines during the allergy process [29]. Expression of these cytokines can result in inhibition, sexual behaviour and food intake [30, 31]. In addition, activation of T-helper type 2-related cytokines can lead to insomnia and sleep loss.

Although all six studies have provided evidence of an association between a form of suicidal behaviour and asthma, more evidence is needed. The studies varied in the categorisation of measured outcomes (death by suicide, suicide attempt and ideation). Only one study, that reported by GOODWIN and EATON [5], attempted to include all three outcomes within a single study. This included completed suicides, although the size of the study population and consequent number of deaths by suicide were too small to provide a conclusive association. Hence, more studies with a larger population size are needed to address the incidence of completed suicide and asthma. 
In youths, suicide ideation and deaths have been examined but no studies have addressed suicide attempts.

Four out of the six studies limited their investigations to crosssectional data, which does not provide information on the sequence of the onset of disorders. The study by GoODWIN and EATON [5] consisted of three time waves providing a follow-up in order to provide a longitudinal prospective. This approach showed a link between suicide ideation and attempts; however, this link was not statistically evident and varied in strength with differing time-periods. The authors attribute this to a small sample size. QIN et al. [26] used population data found in hospital registries. This provided a longitudinal insight; however, data was only available only from when the registry data became available, hence the whole lifetime data for older subjects were not obtained. The cross-sectional design of the other studies did not allow the sequence of outcomes in relation to exposure dates to be measured.

All studies made adjustments for socio-demographic factors, but they varied in adjustments for other confounding effects. Mental disorders, the presence of major depression and smoking were the main confounders considered. GoODWIN and EATON [5] considered asthma treatment and investigated whether treatment of asthma was associated with increased likelihood of suicide ideation and attempt. However, information on specific forms of asthma treatment, as well as frequency and timing of treatment initiation, was limited. Not all studies had measured baseline depression and anxiety. Conclusions regarding the impact of medication use on the risk of suicide ideation and attempts could not be drawn from the data.

Asthma varies in severity and can often lead to hospitalisation. It is thought that depression, anxiety and other adverse psychological factors increase with asthma severity, particularly if the patient is admitted to hospital [24, 32-34]. QIN et al. [26] attempted to address different severities of asthma and allergies by examining completed suicides in patients in both outpatient and in-patient settings. There was no evidence of a significant risk of suicide in patients with allergy rhinitis and bronchial asthma who had outpatient appointments. In the other studies, evidence in the literature does not account for different severities of asthma when examining an association with suicidal behaviour, which is a potential confounder. Other possible confounders not accounted for include genetic and environmental factors, such as the occurrence of an adverse life event. The possible relationship between conditions such as attention deficit hyperactivity disorder and atopic diseases (asthma and rhinitis eczema) is another potential factor to consider in studies investigating suicide-related adverse events [35-39]. Asthma may potentially affect the quality of life experienced by youths and adults in several ways: psychological (self-esteem, social participation or parental over protection); growth and development (short stature or delayed puberty); comorbidity with clinical levels of anxiety and depression [40].

\section{Methodological limitations of previous studies}

Lack of standard definitions for suicide-related behaviour remains a methodological problem when comparing studies, despite attempts to form a consensus. "Suicidality" is not thought to be clinically useful and more specific terminology, such as suicide ideation or suicide attempts, are preferred [41]. In the studies identified in this review, there was no indication of how investigators diagnosed and measured suicide ideation. The questions with regard to suicide asked by interviewers varied between studies. Despite advancements in the definition and nomenclature of suicide-related behaviours, limitations exist due to measurement variability.

All studies were limited by their method of data collection. Asthma diagnosis was determined by self-reporting made by subjects and/or their parents with the exceptions of the studies by GOODWIN et al. [25] and QIN et al. [26], in which asthmatic diagnosis was obtained from data recorded as International Classification of Diseases codes for the purpose of billing patients and a hospital database, respectively. No objective respiratory measures or data on lung function (e.g. spirometry) were taken. Self-reporting of asthma diagnosis may provide a source of recall bias. However, GOODWIN and MARUSIC [23] comment that the method used to determine international prevalence rates of asthma is widely used and accepted, making it comparable to other national and cross-national studies.

Data of deaths by suicide were collected by linking patients to the Department of health death certification system in Taiwan and the National Death Index in the USA in the studies by KUO et al. [10] and GOODWIN and EATON [5], respectively. A limitation of this is that other causes of death (e.g. accidental) may have been substituted for suicide in records to avoid any potential stigma associated with suicide.

This review highlights the need for further well-designed epidemiological studies that investigate the association of asthma and suicide-related adverse events. The use of databases with anonymised electronic patient records can provide large datasets, often representing the general population, and thus is suitable for detecting rare events such as suicide.

\section{Conclusion}

Studies investigating the association between suicide behaviour and asthma support the hypothesis of an association. However, studies with more objective measures adjusting for a wider variety of confounders including medication, measuring all suicide-related outcomes and varying exposure levels (severity of asthma), and taking into account the sequence of diagnosis (longitudinal design) are needed for a more conclusive answer. More evidence with larger population sizes is needed to investigate the association of death by suicide and asthma.

\section{STATEMENT OF INTEREST}

None declared.

\section{REFERENCES}

1 World Health Organization. Prevention of Suicide: Guidelines for the Formulation and Implementaion of National Strategies. Geneva, World Health Organization, 1996.

2 Office for National Statistics. Statistical Bulletin. Suicide rates in the United Kingdom 2000-2009. Newport, Office for National Statistics, 2011.

3 Griffiths C, Roony C, Brock A. Leading causes of death in England and Wales - how should we group causes? Health Stat Q 2005; 6-17. 
4 Nock MK, Borges G, Bromet EJ, et al. Suicide and suicidal behaviour. Epidemiol Rev 2008; 30: 133-154.

5 Goodwin RD, Eaton WW. Asthma, suicidal ideation, and suicide attempts: findings from the Baltimore epidemiologic catchment area follow-up. Am J Public Health 2005; 95: 717-722.

6 Druss B, Pincus H. Suicidal ideation and suicide attempts in general medical illnesses. Arch Intern Med 2000; 160: 1522-1526.

7 Goodwin RD, Kroenke K, Hoven CW, et al. Major depression, physical illness, and suicidal ideation in primary care. Psychosom Med 2003; 65: 501-505.

8 Goodwin RD, Marusic A, Hoven CW. Suicide attempts in the United States: the role of physical illness. Soc Sci Med 2003; 56: 1783-1788.

9 Harris EC, Barraclough BM. Suicide as an outcome for medical disorders. Medicine (Baltimore) 1994; 73: 281-296.

10 Kuo CJ, Chen VC, Lee WC, et al. Asthma and suicide mortality in young people: a 12-year follow-up study. Am J Psychiatry 2010; 167: 1092-1099.

11 Asthma UK. For journalists: key facts and statistics. www.asthma. org.uk/news_media/media_resources/for_journalists_key.html Date last updated: May 27, 2011. Date last accessed: June 1, 2011.

12 Asthma UK. Where do we stand? Asthma in the UK today. London, Asthma UK, 2004.

13 World Health Organization. Asthma. Fact sheet No. 307. Geneva, World Health Organization, 2011. www.who.int/mediacentre/ factsheets/fs307/en/index.html

14 Goodwin RD, Fergusson DM, Horwood LJ. Asthma and depressive and anxiety disorders among young persons in the community. Psychol Med 2004; 34: 1465-1474.

15 Goodwin RD, Messineo K, Bregante A, et al. Prevalence of probable mental disorders among paediatric asthma patients in an inner-city clinic. J Asthma 2005; 42: 643-647.

16 Katon W, Lozano P, Russo J, et al. The prevalence of DSM-IV anxiety and depressive disorders in youth with asthma compared with controls. J Adolesc Health 2007; 41: 455-463.

17 Gangwisch JE, Jacobson CM. New perspectives on assessment of suicide risk. Curr Treat Options Neurol 2009; 11: 371-376.

18 Philip G, Hustad CM, Malice MP, et al. Analysis of behaviourrelated adverse experiences in clinical trials of montelukast. J Allergy Clin Immunol 2009; 124, 699-706: e8.

19 Reith DM, Edmonds L. Assessing the role of drugs in suicidal ideation and suicidality. CNS Drugs 2007; 21: 463-472.

20 Farberow L, McKelligott JW, Cohen S, et al. Suicide among patients with cardiorespiratory illnesses. JAMA 1966; 195: 422-428.

21 Kraft B, Countryman FW, Blumenthal DL. Suicide by asthma. Ann Allergy 1959; 17: 394-398.

22 Levitan H. Suicidal trends in patients with asthma and hypertension: a chart study. Psychother Psychosom 1983; 39: 165-170.

23 Goodwin RD, Marusic A. Asthma and suicidal ideation among youth in the community. Crisis 2004; 25: 99-102.

24 Clarke DE, Goodwin RD, Messias EL, et al. Asthma and suicidal ideation with and without suicide attempts among adults in the United States: what is the role of cigarette smoking and mental disorders? Ann Allergy Asthma Immunol 2008; 100: 439-446.
25 Goodwin RD, Olfson M, Shea S, et al. Asthma and mental disorders in primary care. Gen Hosp Psychiatry 2003; 25: 479-483.

26 Qin P, Mortensen PB, Waltoft BL, et al. Allergy is associated with suicide completion with a possible mediating role of mood disorder - a population-based study. Allergy 2011; 66: 658-664.

27 Goodman SH, Hoven CW, Narrow WE, et al. Measurement of risk for mental disorders and competence in a psychiatric epidemiologic community survey: the National Institute of Mental Health methods for the epidemiology of child and adolescent mental disorders (MECA) study. Soc Psychiatry Psychiatr Epidemiol 1998; 33: 162-173.

28 Lahey BB, Flagg EW, Bird HR, et al. The NIMH methods for the epidemiology of child and adolescent mental disorders (MECA) study: background and methodology. J Am Acad Child Adolesec Psychiatry 1996; 35; 855-864.

29 Galli SJ, Tsai M, Piliponsky AM. The development of allergic inflammation. Nature 2008; 454: 445-454.

30 Kay AB. Allergy and allergic diseases. Second of two parts. N Engl J Med 2001; 344: 109-113.

31 Craig TJ, Ferguson BJ, Krouse JH. Sleep impairment in allergic rhinitis, rhinosinusitis, and nasal polyposis. Am J Otolaryngol 2008; 29: 209-217.

32 Akcakaya N, Aydogan M, Hassanzadeh A, et al. Psychological problems in Turkish asthmatic children and their families. Allergol Immunopathol (Madr) 2003; 31: 282-287.

33 Schneider A, Biessecker K, Quinzler R, et al. Asthma patients with low perceived burden of illness: a challenge for guideline adherence. J Eval Clin Pract 2007; 13: 846-852.

34 Silver EJ, Warman KL, Stein RE. The relationship of caretaker anxiety to children's asthma morbidity and acute care utilization. J Asthma 2005; 42: 379-383.

35 Gibejova A, Mrazek F, Subrtova D, et al. Expression of macrophage inflammatory protein-3 beta/CCL19 in pulmonary sarcoidosis. Am J Respir Crit Care Med 2003; 167: 1695-1703.

36 Vondra V, Prazakova J, Reisova M, et al. Epidemiologie bronchiálního astmatu a bronchiální hyperreaktivity [Epidemiology of bronchial asthma and bronchial hyperreactivity]. Cas Lek Cesk 1990; 129: 714-718.

37 Baker R, Fraser RC, Stone M, et al. Randomised controlled trial of the impact of guidelines, prioritized review criteria and feedback on implementation of recommendations for angina and asthma. $\mathrm{Br}$ J Gen Pract 2003; 53: 284-291.

38 Twena DM, Marshall JS, Haeney MR, et al. A survey of nonatopic and atopic children and adults for the presence of anti-IgE autoantibodies. Clin Immunol Immunopathol 1989; 53: 40-51.

39 Schmitt J, Buske-Kirschbaum A, Roessner V. Is atopic disease a risk factor for attention-deficit/hyperactivity disorder? A systematic review. Allergy 2010; 65: 1506-1524.

40 Katon W. Asthma, suicide risk, and psychiatric comorbidity. Am J Psychiatry 2010; 167: 1020-1022.

41 Meyer RE, Salzman C, Youngstrom EA, et al. Suicidality and risk of suicide-definition, drug safety concerns, and a necessary target for drug development: a consensus statement. J Clin Psychiatry 2010; 71: e1-e21. 